\title{
Effort Complementarity and Sharing Rules in Group
}

\section{Contests*}

\author{
Katsuya Kobayashi $^{\dagger} \quad$ Hideo Konishi ${ }^{\ddagger}$
}

April 10, 2020

\begin{abstract}
In this paper, we consider a prize-sharing rule design problem in a group contest with effort complementarities within groups by employing a CES effort aggregator function. We derive the conditions for a monopolization rule that dominates an egalitarian rule if the objective of the rule design is to maximize the group's winning probability. We find conditions under which the monopolization rule maximizes the group's winning probability, while the egalitarian rule is strictly preferred by all members of the group. Without effort complementarity, there cannot be such a conflict of interest.
\end{abstract}

Keywords: group contest, complementarity in efforts, free riding, prize-sharing rule JEL Classification Numbers: C72, D23, D74

*We thank Editor François Maniquet, Associate Editor, and two anonymous referees for their helpful comments and suggestions. Special thanks are due to Kaoru Ueda for his valuable comments on an earlier version of the paper. This paper was completed when Kobayashi was visiting Boston College on his sabbatical. Kobayashi thanks Hosei University for their financial support and Boston College for their hospitality.

${ }^{\dagger}$ Email katsuyak@hosei.ac.jp, Faculty of Economics, Hosei University, 4342 Aihara-machi, Machida-shi, Tokyo 194-0298, Japan.

‡Email hideo.konishi@bc.edu, Department of Economics, Boston College, 140 Commonwealth Ave., Chestnut Hill, MA 02467 USA. 


\section{Introduction}

Consider multiple R\&D groups competing for a monetary prize. The prize is given to the winner of an R\&D race, and a group's winning probability increases with the group's aggregate effort and decreases with other groups' aggregate efforts. The prize is distributed among the winning group members according to the group's prize-sharing rule. Group members decide how much effort to make based on the prize-sharing rule, while a group leader is a manager who only chooses a prize-sharing rule. The group leader maximizes the winning probability of the group, since he/she has a better chance of promotion after winning the race. Group members are homogenous, and each member's effort level is unobservable (moral hazard) or not contractable by the group leader in designing a prize-sharing rule. This is a stylized group contest model that has been studied in the literature.

Assuming perfectly substitutable individual efforts in each group, Nitzan and Ueda (2014) point out that sharing a winning prize equally among homogeneous group members (an egalitarian rule) does not necessarily maximize the winning probability for the group, even if members' effort cost function is increasing and convex. This is partly because each group member receives a small share of the prize under the egalitarian rule, and partly because of the free-riding incentive. Giving the entire prize to a single member of the group (a monopolization rule) may be better for the winning probability, since it eliminates these two disadvantages, even though it increases the marginal effort cost. Specifically, they find that the monopolization rule maximizes the group's winning probability if (i) the members' marginal effort cost is positive and bounded below, ${ }^{1}$ or (ii) the members' marginal effort cost function starts from zero and increases but is concave. ${ }^{2}$ Moreover, Nitzan and Ueda (2014) find that their condition (ii)

\footnotetext{
${ }^{1}$ This is called PNC (potential non-contributor condition) in Nitzan and Ueda (2014). In addition, this result needs a mild technical condition called RC (regularity condition) for the cost function around zero effort.

${ }^{2}$ They also show that the egalitarian sharing rule maximizes the winning probability if the marginal cost starts from zero and is convex (their anti-Olson theorem).
} 
is identical to that where a group's winning probability goes down as the population of the group goes up. This so-called "group size paradox" was first suggested by Olson (1965), and Esteban and Ray (2001) identify the conditions necessary for it to occur. ${ }^{3}$ Epstein and Mealem (2009) consider a group-specific public-good prize model with heterogeneous valuations among members, but they also come up with a closely related observation.

This paper introduces effort complementarity in group contests to analyze how a sharing rule affects the winning probability of the group as well as the welfare of the group members. To do this, we use a CES effort aggregator function, following Kolmar and Rommeswinkel (2013), where we parameterize the level of complementarity in a simple manner. Complementarity in effort aggregation reduces free-riding incentives, which may make the egalitarian rule more effective. Indeed, we obtain a variation of condition (ii) from Nitzan and Ueda (2014), but the monopolization rule may maximize the winning probability with effort complementarity even if the members' marginal cost function is convex. We will identify the exact condition for this (Propositions 1 and 2). Choi, Chowdhury, and Kim (2016) and Cheikbossian and Fayat (2018) also show related results using CES effort aggregator functions. ${ }^{4}$

More significantly, in the presence of effort complementarity, we show that there may be a conflict of interest between a group leader-who wishes to maximize the winning probability of the group - and the group members - who care about their own payoffs. That is, we show that there is a situation where the egalitarian rule Pareto-dominates the monopolization rule for all group members, while the monopolization rule dominates the egalitarian rule from the

\footnotetext{
${ }^{3}$ Esteban and Ray (2001) clarify that Olson's (1965) results do not depend on whether the prize is public or private. They explain that after fixing the equal allocation of the private good among all members in a group, whether its winning probability increases or not depends on the elasticity of the marginal effort cost when the population increases.

${ }^{4}$ Choi et al. (2016) analyze a two-group contest when the prize is allocated by an intra-group contest between the group members with asymmetric powers. Cheikbossian and Fayat (2018) analyze a contest between two groups by introducing a rivalry parameter of an impure public good prize.
} 
standpoint of the group's winning probability. This happens when (i) there exists moderate effort complementarity and (ii) the marginal effort cost is moderately concave. This result cannot be obtained without effort complementarity.

The remainder of the paper is organized as follows. Section 2 presents our model. In Section 3, we show that the results in Esteban and Ray (2001) and Nitzan and Ueda (2014) still extend in the presence of effort complementarity. Section 4 shows our main result that a group leader and his/her group members may have a conflict of interest with effort complementarity. Section 5 explains how our results for Nash equilibrium in the intragroup game can be extended to an equilibrium analysis in a group contest game, and proves the existence and uniqueness of the equilibrium. Section 6 concludes by discussing the importance of effort complementarity and commenting on a model in Epstein and Mealem (2009), the details of which are given in Appendix B. All proofs are collected in Appendix A.

\section{The Model}

We consider a contest in which $m \geq 2$ groups compete for a prize, focusing on a representative group $i=1,2, \ldots, m$. The population of group $i$ is denoted by $n_{i} \geq 1$. Group members choose their effort levels $e_{i j}, j=1,2, \ldots, n_{i}$, which contribute to their group's winning probability, simultaneously and non-cooperatively. Group members' efforts are aggregated by the CES function of $X_{i}=\left(\sum_{j=1}^{n_{i}} e_{i j}^{r}\right)^{\frac{1}{r}}$, where $r \in(0,1]$ indicates the degree of the effort complementarity. ${ }^{5}$ This CES aggregator function becomes a linear function (perfect substitutes-no complementarity) when $r=1$ and a Cobb-Douglas function when $r=0$ in the limit (each member's effort is essential in the sense that if a member makes no effort then the aggregate

\footnotetext{
${ }^{5}$ Kolmar and Rommeswinkel (2013) are the first in the literature to introduce group members' effort complementarity in group contests by using a CES production function. They call this CES function a group impact function.
} 
effort is also zero).

The winning probability of group $i$ is described as a contest success function $P_{i}=\frac{X_{i}}{X_{i}+X_{-i}},{ }^{6}$ where $X_{-i}=\sum_{k \neq i} X_{k}$ is the other groups' aggregated effort levels. The prize comprises divisible private goods that are shared among members of the winning group, and the value of the prize is normalized to 1 . We denote the share of member $j$ in group $i$ by $a_{i j} \in[0,1]$ and group $i$ 's (prize) sharing rule by $a_{i}=\left(a_{i 1}, \ldots, a_{i n_{i}}\right)$ with $\sum_{j=1}^{n_{i}} a_{i j}=1$. The group leader cannot observe each member's effort or an aggregated group effort. ${ }^{7}$ We assume that group $i$ 's prizesharing rule is chosen by the group leader before each member decides his/her effort level. The effort cost function is common to all members with a constant elasticity $\beta \geq 1$; i.e., member $j$ 's effort cost in group $i$ is described by $\frac{1}{\beta} e_{i j}^{\beta}$. We assume that at least either $r \leq 1$ or $\beta \geq 1$ is a strict inequality. The expected payoff for member $j$ in group $i$ is $U_{i j}=P_{i} a_{i j}-\frac{1}{\beta} e_{i j}^{\beta}$. We assume that all of the above is common knowledge among all players.

Each member in a group decides his/her effort level to maximize his/her expected payoff. We assume that group $i$ members regard the other groups' aggregate effort $X_{-i}$ as given and consider a Nash equilibrium of group $i$ 's effort contribution game as their best response to the other groups' aggregate effort $X_{-i} \cdot{ }^{8}$ Then, the first-order condition of any member $j$ in group $i$ is

$$
\frac{\partial U_{i j}}{\partial e_{i j}}=\frac{\left(\sum_{j=1}^{n_{i}} e_{i j}^{r}\right)^{\frac{1}{r}-1} e_{i j}^{r-1} X_{-i}}{\left(\left(\sum_{j=1}^{n_{i}} e_{i j}^{r}\right)^{\frac{1}{r}}+X_{-i}\right)^{2}} a_{i j}-e_{i j}^{\beta-1}=0 .
$$

This can be rewritten as

$$
P_{i}\left(1-P_{i}\right) \frac{e_{i j}^{r}}{X_{i}^{r}} a_{i j}-e_{i j}^{\beta}=0 .
$$

With these first-order conditions (1), we can investigate how the sharing rule $a_{i}$ affects the members' equilibrium effort levels $\left(e_{i 1}, \ldots, e_{i n_{i}}\right)$ in an effort contribution game in group $i$.

\footnotetext{
${ }^{6}$ We employ the Tullock-form contest success function (Tullock 1980).

${ }^{7}$ Nitzan and Ueda (2011) assume that individual effort levels are observable by the group leader and analyze the case of the convex combination between the egalitarian rule and a relative-effort sharing rule, which allocates the winning prize proportionally to their effort levels.

${ }^{8}$ Baik (2008) calls this Nash equilibrium a group- $i$-specific equilibrium.
} 
From (1), we have $e_{i j}^{r}=\left(\frac{P_{i}\left(1-P_{i}\right)}{X_{i}^{r}}\right)^{\frac{r}{\beta-r}} a_{i j}^{\frac{r}{\beta-r}}$. Summing up all $e_{i j}^{r}$ in group $i$, we have

$$
X_{i}^{r}=\left(\frac{P_{i}\left(1-P_{i}\right)}{X_{i}^{r}}\right)^{\frac{r}{\beta-r}} \sum_{j=1}^{n_{i}} a_{i j}^{\frac{r}{\beta-r}} .
$$

We first raise this expression to the power of $\frac{1}{r}$, then multiply both sides by $X_{i}^{\frac{r}{\beta-r}}$, and finally raise to the power of $\beta-r$. Following this, the aggregate effort $X_{i}$ at a Nash equilibrium in group $i$ can be implicitly described as

$$
X_{i}^{\beta}=P_{i}\left(1-P_{i}\right) A_{i}
$$

where $A_{i}=\left(\sum_{j=1}^{n_{i}} a_{i j}^{\frac{r}{\beta-r}}\right)^{\frac{\beta-r}{r}}$ can be viewed as group $i$ 's productivity, which affects its winning probability positively.

\section{Sharing rules that maximize winning probability}

Our first question is which sharing rule would maximize the winning probability of group $i$ in Nash equilibrium given $X_{-i}$. Thanks to our functional specifications of the cost and effort aggregator functions, we can clearly relate $X_{i}$ to prize-sharing rules, despite the added effort complementarity in the model.

Lemma 1. In Nash equilibrium in group $i$, the equilibrium winning probability of group $i$ increases in $A_{i}$. That is, group $i$ 's winning probability is maximized by a sharing rule $\left(a_{i j}\right)_{j=1}^{n_{i}}$ that maximizes $A_{i}$.

Since group members are homogenous and their efforts are unobservable, the following two sharing rules play important roles among all sharing rules $\left(a_{i j}\right)_{j=1}^{n_{i}}$ : the egalitarian rule that allocates the prize equally among all group members $\left(a_{i j}=1 / n_{i}\right.$ for all member $\left.j\right)$, and the monopolization rule that allocates the entire prize to an arbitrarily chosen group member (members are homogenous), leaving nothing for the rest $\left(a_{i j}=1\right.$ for some member $j$, and 
$a_{i l}=0$ for any other member $l \neq j$ ). The next proposition shows that these two rules maximize the winning probability of group $i$, depending on $r$ and $\beta .^{9}$

Proposition 1. When $2 r<\beta$, the egalitarian rule maximizes the winning probability of group $i$. When $2 r>\beta$, the monopolization rule maximizes the winning probability of group $i$. When $2 r=\beta$, the winning probability of group $i$ is the same under any sharing rules.

We can interpret this result in the context of $R \& D$ competition. Some R\&D projects benefit from coordinated efforts (strong effort complementarity: small $r$ ), while others do not (weak effort complementarity: large $r$ ). Proposition 1 says that the group leader should choose the egalitarian rule for projects with strong effort complementarity $(2 r<\beta)$, since treating everybody equally enhances aggregate effort the most. In contrast, the group leader should use the monopolization rule by selecting a single member for projects with weak complementarity $(2 r>\beta)$, since it eliminates all free-riding incentives and maximizes an incentive for effort by letting the selected member monopolize the prize. If $2 r=\beta$, then $A_{i}$ is the same under any sharing rules $\left(a_{i j}\right)_{j=1}^{n_{i}}$. Nitzan and Ueda (2014) report the above result without effort complementarity ( $r=1$; Proposition 4 in Nitzan and Ueda 2014).

The probabilities under the egalitarian rule and the monopolization rule are denoted by $P_{i E}$ and $P_{i M}$, respectively. Under the egalitarian rule in group $i$, every member's effort is the same, which is denoted by $e_{i}$ in a Nash equilibrium in group $i$. Then $X_{i}=\left(\sum_{j=1}^{n_{i}} e_{i j}^{r}\right)^{\frac{1}{r}}=\left(n_{i} e_{i}^{r}\right)^{\frac{1}{r}}=$ $n_{i}^{\frac{1}{r}} e_{i}$. Thus, (1) becomes

$$
e_{i}^{\beta}=P_{i E}\left(1-P_{i E}\right) \frac{1}{n_{i}^{2}} .
$$

Since (2) implies $e_{i}=n_{i}^{-\frac{2}{\beta}} P_{i E}^{\frac{1}{\beta}}\left(1-P_{i E}\right)^{\frac{1}{\beta}}$, when we substitute this into the definition of $P_{i}$, we

\footnotetext{
${ }^{9}$ In a group contest for multiple (homogenous) indivisible prizes, Crutzen, Flamand, and Sahuguet (2020) obtain a closely related result. When $2 r<\beta$, allocating prizes according to a fair lottery maximizes group success, while when $2 r>\beta$, allocating prizes according to a predetermined (priority) list is better than the fair lottery allocation rule.
} 
are able to solve $P_{i E}$ as a function of parameters, in particular, $n_{i}$ and $r$ implicitly:

$$
P_{i E}\left(n_{i}, r\right)=\frac{n_{i}^{\frac{1}{r}} e_{i}}{n_{i}^{\frac{1}{r}} e_{i}+X_{-i}}=\frac{n_{i}^{\frac{\beta-2 r}{r \beta}} P_{i E}\left(n_{i}, r\right)^{\frac{1}{\beta}}\left(1-P_{i E}\left(n_{i}, r\right)\right)^{\frac{1}{\beta}}}{n_{i}^{\frac{\beta-2 r}{r \beta}} P_{i E}\left(n_{i}, r\right)^{\frac{1}{\beta}}\left(1-P_{i E}\left(n_{i}, r\right)\right)^{\frac{1}{\beta}}+X_{-i}} .
$$

Each member's payoff, denoted by $U_{i E}$, then becomes

$$
U_{i E}\left(n_{i}, r\right)=P_{i E}\left(n_{i}, r\right) \frac{1}{n_{i}}-\frac{1}{\beta} e_{i}^{\beta}=\frac{P_{i E}\left(n_{i}, r\right)}{n_{i}}\left(1-\frac{1}{\beta}\left(1-P_{i E}\left(n_{i}, r\right)\right) \frac{1}{n_{i}}\right)
$$

using (3).

Under the monopolization rule, on the other hand, the effort level and payoff of the monopolizing member are equal to the above expressions of (3) and $U_{i E}$ in the case of $n_{i}=1$, since the other members for whom nothing is allocated to exert no effort. The payoff of the member exerting effort is denoted by $U_{i M}$. His/her payoff is

$$
U_{i M}=P_{i M}\left(1-\frac{1-P_{i M}}{\beta}\right)
$$

By setting $n_{i}=1$ in (4), the winning probability under the monopolization rule is

$$
P_{i M}=\frac{P_{i M}^{\frac{1}{\beta}}\left(1-P_{i M}\right)^{\frac{1}{\beta}}}{P_{i M}^{\frac{1}{\beta}}\left(1-P_{i M}\right)^{\frac{1}{\beta}}+X_{-i}} .
$$

Note that $P_{i M}$ and $U_{i M}$ do not depend on $r$, and that $P_{i E}(1, r)=P_{i M}$ and $U_{i E}(1, r)=U_{i M}$.

We first prove the following lemma for the winning probability of group $i$ under the egalitarian rule.

Lemma 2. We have the following comparative static results:

$$
\frac{d P_{i E}}{d r}=\frac{\log n_{i}}{r^{2}} \frac{P_{i E}\left(1-P_{i E}\right) \beta}{1-2 P_{i E}-\beta}<0
$$

and

$$
\frac{d P_{i E}}{d n_{i}}=\frac{2 r-\beta}{r n_{i}} \frac{P_{i E}\left(1-P_{i E}\right)}{1-2 P_{i E}-\beta} .
$$

Regarding the group member population, we have the following result, using (6) in Lemma 2. 
Proposition 2. Under the egalitarian rule, the winning probability of group $i$ is strictly increasing, constant, and strictly decreasing in the number of group members $n_{i}$ if and only if $2 r<\beta, 2 r=\beta$, and $2 r>\beta$, respectively.

Esteban and Ray (2001) show the condition necessary to improve the winning probability of a group when its size increases under the egalitarian rule (together with the fixed allocation between private and public rewards). The sign of one minus the elasticity of marginal effort cost in Esteban and Ray (2001) corresponds to $\beta \gtreqless 2$ in our study, since there is no complementarity among members' efforts $(r=1)$ in their case. Epstein and Mealem (2009) provide a nice summary of the results in their equation (12) and the following discussions (page 364 in Epstein and Mealem, 2009). ${ }^{10}$ To sum up these results in tandem with ours, as long as the focus is on winning probability, both the elasticity of the marginal effort cost and the complementarity among group members' efforts are interchangeable.

\section{Conflict of interest between the group leader and mem- bers}

So far, we have been focusing on group $i$ 's winning probability. Our second question is as follows. When $2 r>\beta$, we have seen that it would be best for the winning probability if the prize was monopolized by a single member. However, the member needs to exert the entire group's effort on his/her own in order to win, since the other members get no benefit and exert no effort. Considering the relation between the effort complementarity and the elasticity of the marginal effort cost, it is not clear whether the member who monopolizes the prize prefers the monopolization rule to the egalitarian rule. If the egalitarian rule is better for him/her than the monopolization rule at a specific $r$, then switching from the monopolizing rule to the

\footnotetext{
${ }^{10}$ See also the "anti-Olson theorem" in Nitzan and Ueda (2014).
} 
egalitarian rule may improve every member's payoff even though it might reduce the group's winning probability. In other words, sharing the prize equally with the other members in a group may Pareto-improve the group's welfare. We confirm this as follows by focusing on the case where $2 r>\beta$.

Since $U_{i E}(1, r)=U_{i M}$ for all $r$, if we can show that $U_{i E}\left(n_{i}, r\right)$ is monotonically decreasing in $n_{i}$ for all $n_{i} \geq 1$, we will have $U_{i E}\left(n_{i}, r\right)<U_{i M}$ for $n_{i} \geq 2$. The next lemma shows that this is indeed the case when $r=1$.

Lemma 3. Suppose that $r=1$. Then, we have $\frac{d U_{i E}\left(n_{i}, 1\right)}{d n_{i}}<0$ for all $n_{i} \geq 1$; i.e., $U_{i E}\left(n_{i}, 1\right)<$ $U_{i M}\left(n_{i}, 1\right)=U_{i M}$ for all $n_{i} \geq 2$.

The following lemma shows that there is a threshold value of $r$ for the payoffs under the egalitarian and monopolization rules.

Lemma 4. Suppose that $\beta \leq 2 r, n_{i} \geq 2$, and $1+\frac{1}{n_{i}}>\beta$ hold. If group $i$ 's winning probability is low, in the sense that $1-\frac{n_{i}}{n_{i}+1} \beta>P_{i M}$, then there is a unique $\hat{r} \in\left(\frac{\beta}{2}, 1\right)$, such that $U_{i E}\left(n_{i}, r\right)<$ $U_{i M}$ for all $r \in(\hat{r}, 1]$, and $U_{i E}\left(n_{i}, r\right)>U_{i M}$ for any $r \in\left[\frac{\beta}{2}, \hat{r}\right)$.

This Lemma has an interesting implication. The following proposition shows that there may be conflict between winning probability maximization and Pareto optimality in group $i$.

Proposition 3. Suppose that $\beta<2 r, n_{i} \geq 2$, and $1+\frac{1}{n_{i}}>\beta$ hold. If group i's winning probability is low, in the sense that $1-\frac{n_{i}}{n_{i}+1} \beta>P_{i M},{ }^{11}$ then there is a unique $\hat{r} \in\left(\frac{\beta}{2}, 1\right)$, such that the egalitarian rule Pareto-dominates the monopolization rule for any $r \in\left(\frac{\beta}{2}, \hat{r}\right)$ within

\footnotetext{
${ }^{11}$ In Proposition 3, condition $1-\frac{n_{i}}{n_{i}+1} \beta>P_{i M}$ appears to be a condition for an endogenous variable $P_{i M}$ because $P_{i M}$ is explicitly unsolvable. However, $P_{i M}$ is uniquely led by

$$
P_{i M}=\frac{P_{i M}^{\frac{1}{\beta}}\left(1-P_{i M}\right)^{\frac{1}{\beta}}}{P_{i M}^{\frac{1}{\beta}}\left(1-P_{i M}\right)^{\frac{1}{\beta}}+X_{-i}} .
$$

This is determined by the exogenous variables $\beta$ and $X_{-i}$ only. Thus, we can confirm if condition $1-\frac{n_{i}}{n_{i}+1} \beta>$ $P_{i M}$ is satisfied with economic data $\beta, n_{i}$, and $X_{-i}$.
} 
group $i$, despite the fact that the monopolization rule achieves a higher winning probability than the egalitarian rule. In contrast, if $1-\frac{n_{i}}{n_{i}+1} \beta>P_{i M}$ is not satisfied, then the single member who monopolizes the prize prefers the monopolization rule to the egalitarian rule.

This Pareto dominance of the egalitarian rule is due to the complementarity among group members' efforts. Without the complementarity, this Pareto dominance disappears. In fact, at $r=1$, the Pareto dominance does not hold.

\section{Equilibrium in Group Contest}

We can apply our analysis to show that our group contest model has an equilibrium. We will consider a two-stage game as follows. Stage 1: Each group leader who maximizes the winning probability of his/her group decides its sharing rule simultaneously, and Stage 2: members of all groups simultaneously choose their effort levels. In this paper, we assume that each groups' sharing rules are observable and employ subgame perfect equilibrium as our solution concept. ${ }^{12}$ We can allow for asymmetric groups - different groups can have different $\beta_{i}, r_{i}$, and $n_{i}$. The key is to show that a Nash equilibrium exists and is unique in Stage 2. We show that each group's best response to the aggregation of the other groups' effort levels $X_{-i}$ is at a Nash equilibrium. The effort contribution game of any group $i$ in Stage 2 is described as a function $\psi_{i}\left(X_{-i} ; a_{i}, \beta_{i}, r_{i}\right) \rightarrow X_{i}$. Using the share-function approach (Esteban and Ray 2001, Ueda 2002, and Cornes and Hartley 2005), ${ }^{13}$ we can guarantee the existence and uniqueness of the Nash equilibrium by each $\psi_{i}$ 's continuity and strict monotonicity in $X_{-i}$.

\footnotetext{
${ }^{12}$ Readers may think that it is unrealistic to assume that groups can observe other groups' sharing rules. Nitzan and Ueda (2011) assume that sharing rules are the private information of each group and use perfect Bayesian equilibrium with the same beliefs for other groups' sharing rules at every information set. Since the model does not involve a real asymmetric information problem, their perfect Bayesian equilibrium coincides with our subgame perfect equilibrium under complete information.

${ }^{13}$ We thank Kaoru Ueda for suggesting that we use the share function approach.
} 
Let $X=X_{i}+X_{-i}$. By using $P_{i}=\frac{X_{i}}{X}$ and reformulating (2), $P_{i}$, which is regarded as group $i$ 's share function, is described as

$$
P_{i}^{1-\beta_{i}}\left(1-P_{i}\right) A_{i}=X^{\beta_{i}}
$$

for all group $i=1, \ldots, m$. By totally differentiating the above, we obtain

$$
A_{i} P_{i}^{-\beta_{i}}\left(1-\beta_{i}-\left(2-\beta_{i}\right) P_{i}\right) d P_{i}+P_{i}^{1-\beta_{i}}\left(1-P_{i}\right) d A_{i}-\beta_{i} X^{\beta_{i}-1} d X=0 .
$$

Therefore, we have

$$
\frac{\partial P_{i}}{\partial X}=\frac{\beta_{i} P_{i}\left(1-P_{i}\right)}{\left(1-\beta_{i}-\left(2-\beta_{i}\right) P_{i}\right) X}
$$

by using $A_{i}=X^{\beta_{i}} /\left(P_{i}^{1-\beta_{i}}\left(1-P_{i}\right)\right)$ from $(7)$. We have also

$$
\frac{\partial P_{i}}{\partial A_{i}}=\frac{-P_{i}\left(1-P_{i}\right)}{\left(1-\beta_{i}-\left(2-\beta_{i}\right) P_{i}\right) A_{i}} .
$$

Note that the denominators of (8) and (9) are negative if $\beta_{i} \leq 2$ (since $\beta_{i} \geq 1$ ), and are also negative if $\beta_{i}>2$ (since $\left.P_{i} \leq 1\right)$. Thus, $\partial P_{i} / \partial X<0$ holds for any $X$ and $A_{i}$ in (8). From (7), we have $\lim _{X \rightarrow 0} P_{i}\left(X ; A_{i}\right)=1, \lim _{X \rightarrow \infty} P_{i}\left(X ; A_{i}\right)=0$, and $P_{i}\left(X ; A_{i}\right)$ is continuous. These facts hold for all $i=1, \ldots, m$.

A vector of sharing rules $\left(A_{1}, \ldots, A_{m}\right)$ is described as $A$. Let the aggregate share function $f: \mathbb{R}_{++} \rightarrow \mathbb{R}$ be such that $f(X ; A)=\sum_{i=1}^{m} P_{i}\left(X ; A_{i}\right)$. From the above facts regarding $P_{i}\left(X ; A_{i}\right), f(X ; A)$ is continuous and strictly decreases with $X$ by $\lim _{X \rightarrow 0} f(X ; A)=m$ and $\lim _{X \rightarrow \infty} f(X ; A)=0$. Therefore, there is a unique $X^{*}$ at which $f\left(X^{*} ; A\right)=\sum_{i=1}^{m} P_{i}\left(X^{*} ; A_{i}\right)=$ 1, and there is a unique Nash equilibrium for any arbitrary $A$ in Stage 2 .

Proposition 4. For any profile of group sharing rules $\left(a_{1}, \ldots, a_{m}\right)$ with $a_{i}=\left(a_{i 1}, \ldots, a_{i n_{i}}\right)$ for all $i=1, \ldots, m$, there is a unique Nash equilibrium in the effort choice stage.

Since (9) is positive, the $P_{i}\left(X ; A_{i}\right)$ function shifts up everywhere as $A_{i}$ increases. Then, the aggregate share function $f(X ; A)$ shifts up in turn. Thus, an increase in $A_{i}$ raises group $i$ 's probability of winning, together with the total effort level $X$ at Nash equilibrium in Stage 2. 
This implies that each group leader's objective is to maximize his/her $A_{i}$ in Stage 1 , which is the same result as Lemma 1.

Lemma 5. In Stage 1 of the group contest game, the equilibrium winning probability of group $i$ is increasing in $A_{i}$. That is, group $i$ 's winning probability is maximized by a sharing rule $\left(a_{i j}\right)_{j=1}^{n_{i}}$ that maximizes $A_{i}$.

This lemma leads us to a counterpart of Proposition 1-that is, the results of Proposition 1 are valid in the two-stage group contest game.

Proposition 5. In Stage 1 of the group contest game, each group i's leader chooses its sharing rule to maximize the winning probability $P_{i}$ as follows: (i) use the egalitarian rule if $2 r_{i}<\beta_{i}$, (ii) use the monopolization rule ${ }^{14}$ if $2 r_{i}>\beta_{i}$, and (iii) use any sharing rule if $2 r_{i}=\beta_{i}$.

A corollary of this proposition is that there is an essentially unique subgame perfect equilibrium in our group contest game, since each group leader's strategy is solely dependent on $2 r_{i} \lesseqgtr \beta_{i}$

Corollary 1. For all $\left(r_{i}, \beta_{i}\right)_{i=1}^{m}$, groups' equilibrium winning probabilities $\left(P_{i}\right)_{i=1}^{m}$ are uniquely determined.

The results of Proposition 5 depend only on the exogenous variables of $r_{i}$ and $\beta_{i}$. Thus, Proposition 5 and Corollary 1 indicate that Proposition 3 is also valid at the subgame perfect equilibrium in the two-stage group contest game. That is, for some group $i$, when $2 r_{i}>\beta_{i}$ and $1+\frac{1}{n_{i}}>\beta_{i}$ under the asymmetric parameters, if $1-\frac{n_{i}}{n_{i}+1} \beta_{i}>P_{i M}$ holds, then there is a conflict of interest between the group leader and his/her group members at the subgame perfect equilibrium. If $1-\frac{n_{i}}{n_{i}+1} \beta_{i}>P_{i M}$ is violated, then the monopolizing member has an incentive to work with the group leader, since it is in their common interests to choose the

\footnotetext{
${ }^{14}$ When the group leader chooses the monopolization rule at Stage 1, effort complementarity is irrelevant on the equilibrium path. Effort complementarity is in effect only off the equilibrium path.
} 
monopolization rule and exclude the rest of the group. In addition, if the parameters are symmetric, the condition is simply described as the relation among the number of groups, the group population, and the elasticity of the marginal effort cost. In this case, since $P_{i M}$ becomes $\frac{1}{m}$, the condition is $1-\frac{n_{i}}{n_{i}+1} \beta>\frac{1}{m}$.

\section{Concluding remarks}

We conclude our paper by commenting on Epstein and Mealem (2009). They use a generalized Tullock contest by introducing power $r \in[0,1]$ : i.e., $X_{i}=\sum_{j=1}^{n_{i}} e_{i j}^{r}$. This form may look

similar to our CES form, $X_{i}=\left(\sum_{j=1}^{n_{i}} e_{i j}^{r}\right)^{\frac{1}{r}}$, and readers may wonder if our Proposition 3 may hold in their case. It turns out that their generalized Tullock contest cannot generate conflicts of interest between the group leader and his/her group members - we can confirm that with their form, the egalitarian rule's Pareto dominance in Proposition 3 cannot occur. Thus, effort complementarity is essential in getting our conflict-of-interest result. In contrast, with their contest success function, our Propositions 1 and 2 hold. We detail the analysis in Appendix B.

\section{Appendix A}

Here, we collect all proofs.

Proof of Lemma 1. Recalling $X_{i}=\left(\sum_{j=1}^{n_{i}} e_{i j}^{r}\right)^{\frac{1}{r}}$ and given $X_{-i}$, maximizing the winning probability of group $i$ means that $X_{i}$ becomes as large as possible at Nash equilibrium in group $i$. If $X_{i}$ is a strictly increasing function of $A_{i}$, we can maximize $X_{i}$ by maximizing $A_{i}$ subject to $\sum_{j=1}^{n_{i}} a_{i j}=1$.

From (2), let $\phi\left(X_{i}, A_{i}\right)=X_{i}^{\beta}-P_{i}\left(1-P_{i}\right) A_{i}=0$. Recalling that $\beta \geq 1,0<r \leq 1$ and that $P_{i}$ is a function of $X_{i}$ through $P_{i}=\frac{X_{i}}{X_{i}+X_{-i}}$, and by differentiating $\phi$ with respect to $A_{i}$, we get

$$
\frac{d X_{i}}{d A_{i}}=-\frac{\phi_{A_{i}}}{\phi_{X_{i}}}=\frac{P_{i}\left(1-P_{i}\right)}{X_{i}^{\beta-1}\left(\beta-1+2 P_{i}\right)}>0
$$


for $\beta>r$ by using $A_{i}=\frac{X_{i}^{\beta}}{P_{i}\left(1-P_{i}\right)}$ from (2). Thus, $X_{i}$ is a strictly increasing function in $A_{i}$ for $\beta>r$.

Proof of Proposition 1. From Lemma 1, it is enough to maximize $A_{i}$. It is also enough to maximize the contents in parentheses in $A_{i}$ because $\frac{\beta-r}{r}>0$. Note that $A_{i}^{\frac{r}{\beta-r}}=\sum_{j=1}^{n_{i}} a_{i j}^{\frac{r}{\beta-r}}$ is an additively separable function. Since $r>0$, our maximization problem boils down to

$$
\max \sum_{j=1}^{n_{i}} a_{i j}^{\frac{r}{\beta-r}} \text { subject to (i) } \sum_{j=1}^{n_{i}} a_{i j}=1 \text { and (ii) } a_{i j} \geq 0 \text { for all } j=1, \ldots, n_{i} \text {. }
$$

Thus, it is easy to see that $\frac{r}{\beta-r} \lesseqgtr 1$ dictates the optimal sharing rule. We obtain three cases:

Case 1: If $2 r<\beta, A_{i}$ is maximized when $a_{1}=a_{2}=\ldots=a_{n_{i}}=1 / n_{i}$.

Case 2: If $2 r=\beta, A_{i}$ is constant for any sharing rule.

Case 3: If $2 r>\beta, A_{i}$ is maximized when $a_{i j}=1$ for a single $j$, and $a_{i \ell}=0$ for all other $\ell$.

Proof of Lemma 2. Let $\Delta \equiv n_{i}^{-\frac{\beta-2 r}{r \beta}}$ in relation to $n_{i}$ in (4). Rewriting (4), we have

$$
\left(1-P_{i E}\right)\left(P_{i E}\left(1-P_{i E}\right)\right)^{\frac{1}{\beta}}=P_{i E} X_{-i} \Delta .
$$

By totally differentiating the above, we obtain

$$
\left[-\left(P_{i E}\left(1-P_{i E}\right)\right)^{\frac{1}{\beta}}-X_{-i} \Delta+\frac{1}{\beta}\left(1-P_{i E}\right)\left(P_{i E}\left(1-P_{i E}\right)\right)^{\frac{1}{\beta}-1}\left(1-2 P_{i E}\right)\right] d P_{i E}=P_{i E} X_{-i} d \Delta .
$$

After solving (10) for $X_{-i}$, we substitute it into the above and obtain

$$
\frac{1}{P_{i E}}\left(P_{i E}\left(1-P_{i E}\right)\right)^{\frac{1}{\beta}}\left[-1+\frac{1}{\beta}\left(1-2 P_{i E}\right)\right] d P_{i E}=\left(1-P_{i E}\right)\left(P_{i E}\left(1-P_{i E}\right)\right)^{\frac{1}{\beta}} \frac{d \Delta}{\Delta}
$$

or

$$
\frac{d P_{i E}}{d \Delta}=\frac{P_{i E}\left(1-P_{i E}\right)}{\Delta\left[-1+\frac{1}{\beta}\left(1-2 P_{i E}\right)\right]}
$$

Since $-1+\frac{1}{\beta}\left(1-2 P_{i E}\right)<0$ from $\beta \geq 1$, we have $\frac{d P_{i E}}{d \Delta}<0$. By differentiating $\Delta$ with respect to $n_{i}$ and $r$, we have

$$
\frac{d \Delta}{d n_{i}}=-\frac{\beta-2 r}{r \beta} \times \frac{\Delta}{n_{i}}
$$


and

$$
\frac{d \Delta}{d r}=\frac{1}{r^{2}}\left(\log n_{i}\right) \Delta>0
$$

respectively. We obtain the results using the chain rule.

Proof of Lemma 3. Recall $U_{i E}\left(n_{i}, r\right)=\frac{P_{i E}\left(n_{i}, r\right)}{n_{i}}\left(1-\frac{1}{\beta}\left(1-P_{i E}\left(n_{i}, r\right)\right) \frac{1}{n_{i}}\right) \equiv \tilde{U}\left(n_{i}, P_{i E}\left(n_{i}, r\right)\right)$.

This implies

$$
\frac{\partial \tilde{U}}{\partial P_{i E}}=\frac{1}{n_{i}}-\frac{1}{\beta n_{i}^{2}}\left(1-2 P_{i E}\right)=\frac{1}{n_{i}^{2} \beta}\left(n_{i} \beta+2 P_{i E}-1\right) .
$$

Thus, by totally differentiating $\tilde{U}\left(n_{i}, P_{i E}\left(n_{i}, 1\right)\right)$ with respect to $n_{i}$ using $(6)$, we obtain

$$
\begin{aligned}
& \frac{d U_{i E}\left(n_{i}, 1\right)}{d n_{i}} \\
= & \frac{\partial \tilde{U}}{\partial n_{i}}+\frac{\partial \tilde{U}}{\partial P_{i E}} \frac{d P_{i E}}{d n_{i}} \\
= & \frac{P_{i E}}{n_{i}^{2}}\left[-1+\frac{2}{n_{i} \beta}\left(1-P_{i E}\right)\right]+\frac{(2-\beta) P_{i E}\left(1-P_{i E}\right)}{n_{i}\left(1-2 P_{i E}-\beta\right)}\left[\frac{1}{n_{i}^{2} \beta}\left(n_{i} \beta+2 P_{i E}-1\right)\right] \\
= & \frac{P_{i E}}{n_{i}^{3}}\left[-n_{i}+\frac{2}{\beta}\left(1-P_{i E}\right)+\left(1-P_{i E}\right) \frac{2-\beta}{1-2 P_{i E}-\beta}\left(n_{i}+\frac{1}{\beta}\left(2 P_{i E}-1\right)\right)\right] \\
= & \frac{P_{i E}}{n_{i}^{3} \beta\left(1-2 P_{i E}-\beta\right)} \\
& \times\left[-n_{i} \beta\left(1-2 P_{i E}-\beta\right)+2\left(1-P_{i E}\right)\left(1-2 P_{i E}-\beta\right)+\left(1-P_{i E}\right)(2-\beta)\left(\beta n_{i}-\left(1-2 P_{i E}\right)\right)\right] .
\end{aligned}
$$

Since $1-2 P_{i E}-\beta<0$, we can focus on the sign of the contents of the brackets:

$$
\begin{aligned}
{[\cdot]=} & -n_{i} \beta\left(1-2 P_{i E}-\beta\right)+n_{i} \beta\left(2-\beta-2 P_{i E}+P_{i E} \beta\right) \\
& +2\left(1-2 P_{i E}-\beta\right)\left(1-P_{i E}\right)-\left(1-P_{i E}\right)(2-\beta)\left(1-2 P_{i E}\right) \\
= & \beta\left[\left(n_{i}-1\right)+P_{i E}\left(n_{i} \beta-1+2 P_{i E}\right)\right]>0
\end{aligned}
$$

for any $n_{i} \geq 1$. Thus, we conclude that $\frac{d U_{i E}}{d n_{i}}<0$ for any $n_{i} \geq 1$. This implies that $U_{i E}\left(n_{i}, 1\right)<$ $U_{i M}$ for any $n_{i} \geq 2$ when $r=1$. We have completed the proof.

Proof of Lemma 4. First note that the assumptions $n_{i} \geq 2$ and $1+\frac{1}{n_{i}}>\beta$ imply $2>\beta$. 
Consider the case of $r=\frac{\beta}{2}$. Since $P_{i E}\left(n_{i}, \frac{\beta}{2}\right)=P_{i E}\left(1, \frac{\beta}{2}\right)=P_{i M}$ by Proposition 1 , we have

$$
\begin{aligned}
U_{i E}\left(n_{i}, \frac{\beta}{2}\right) & =\frac{P_{i E}\left(n_{i}, \frac{\beta}{2}\right)}{n_{i}}\left(1-\frac{1}{\beta}\left(1-P_{i E}\left(n_{i}, \frac{\beta}{2}\right)\right) \frac{1}{n_{i}}\right) \\
& =\frac{P_{i M}}{n_{i}}\left(1-\frac{1}{\beta}\left(1-P_{i M}\right) \frac{1}{n_{i}}\right) .
\end{aligned}
$$

By subtracting $U_{i M}=P_{i M}\left[1-\frac{1}{\beta}\left(1-P_{i M}\right)\right]$ from $U_{i E}\left(n_{i}, \frac{\beta}{2}\right)$, we obtain

$$
\begin{aligned}
U_{i E}\left(n_{i}, \frac{\beta}{2}\right)-U_{i M} & =P_{i M}\left[-1+\frac{1}{\beta}\left(1-P_{i M}\right)+\frac{1}{n_{i}}\left(1-\frac{1}{\beta}\left(1-P_{i M}\right) \frac{1}{n_{i}}\right)\right] \\
& =P_{i M}\left[-\left(1-\frac{1}{n_{i}}-\frac{1}{\beta}\left(1-\frac{1}{n_{i}^{2}}\right)\right)-\frac{1}{\beta} P_{i M}\left(1-\frac{1}{n_{i}^{2}}\right)\right] \\
& =P_{i M}\left(1-\frac{1}{n_{i}^{2}}\right)\left[-\frac{n_{i}}{n_{i}+1}+\frac{1}{\beta}-\frac{1}{\beta} P_{i M}\right] .
\end{aligned}
$$

Then, the condition of $U_{i E}\left(n_{i}, \frac{\beta}{2}\right)>U_{i M}$ is

$$
1-\frac{n_{i}}{n_{i}+1} \beta>P_{i M}
$$

That is, if (12) is satisfied, $U_{i E}\left(n_{i}, \frac{\beta}{2}\right)>U_{i M}$ holds, while $U_{i E}\left(n_{i}, 1\right)<U_{i M}$. Since $\frac{d P_{i E}}{d r}<0$ holds by (5) in Lemma 2 and from (11), $\frac{\partial \tilde{U}}{\partial P_{i E}}=\frac{1}{n_{i}^{2} \beta}\left(n_{i} \beta+2 P_{i E}-1\right)>0$, we have $\frac{d U_{i E}\left(P_{i E}(r)\right)}{d r}=$ $\frac{\partial \tilde{U}_{i E}}{\partial P_{i E}} \frac{d P_{i E}}{d r}<0$, which is $U_{i E}$ monotonically decreasing in $r$. Considering the above facts and given that $U_{i E}$ is continuous in $r$, there is a unique $\hat{r} \in\left(\frac{\beta}{2}, 1\right)$, such that $U_{i E}\left(n_{i}, r\right)<U_{i M}$ holds for all $r \in(\hat{r}, 1]$ and $U_{i E}\left(n_{i}, r\right)>U_{i M}$ holds for all $r \in\left[\frac{\beta}{2}, \hat{r}\right)$.

Proof of Lemma 5. First, focus on the $P_{i}\left(X ; A_{i}\right)$ function. Starting from the original $A_{i}$ and equilibrium $X^{*}, A_{i}$ is increased by $\Delta A_{i}>0$. Since $\frac{\partial P_{i}}{\partial A_{i}}>0$ for all $X$ from (9), the $P_{i}$ function shifts up vertically. Let $\tilde{X}$ be such that $P_{i}\left(X^{*} ; A_{i}\right)=P_{i}\left(\tilde{X} ; A_{i}+\Delta A_{i}\right)$ (see Figure 1$)$. Since $\frac{\partial P_{i}}{\partial X}<0$ from (8), $\tilde{X}>X^{*}$ holds, and for any $X \in\left(X^{*}, \tilde{X}\right)$, we have $P_{i}\left(X ; A_{i}+\Delta A_{i}\right)>$ $P_{i}\left(X^{*} ; A_{i}\right)$. Recall that the equilibrium $X^{*}$ is described by the aggregate share function

$$
f\left(X^{*} ; A\right)=\sum_{i^{\prime} \neq i} P_{i^{\prime}}\left(X^{*} ; A_{i^{\prime}}\right)+P_{i}\left(X^{*} ; A_{i}\right)=1 .
$$

Let $A_{-i}$ be a vector that removes $A_{i}$ from $A$. By increasing $A_{i}$ by $\Delta A_{i}$, the equilibrium aggregate effort $X^{* *}$ satisfies

$$
f\left(X^{* *} ; A_{i}+\Delta A_{i}, A_{-i}\right)=\sum_{i^{\prime} \neq i} P_{i^{\prime}}\left(X^{* *} ; A_{i^{\prime}}\right)+P_{i}\left(X^{* *} ; A_{i}+\Delta A_{i}\right)=1 .
$$


Since $\frac{\partial P_{i^{\prime}}}{\partial X}<0$ for all $i^{\prime}=1, \ldots, m$, we have $X^{* *}>X^{*}$ and

$$
\begin{aligned}
f\left(\tilde{X} ; A_{i}+\Delta A_{i}, A_{-i}\right) & =\sum_{i^{\prime} \neq i} P_{i^{\prime}}\left(\tilde{X} ; A_{i^{\prime}}\right)+P_{i}\left(\tilde{X} ; A_{i}+\Delta A_{i}\right) \\
& =\sum_{i^{\prime} \neq i} P_{i^{\prime}}\left(\tilde{X} ; A_{i^{\prime}}\right)+P_{i}\left(X^{*} ; A_{i}\right)<1 .
\end{aligned}
$$

By the intermediate value theorem, $X^{* *} \in\left(X^{*}, \tilde{X}\right)$ holds. We conclude $P_{i}\left(X^{* *} ; A_{i}+\Delta A_{i}\right)>$ $P_{i}\left(X^{*} ; A_{i}\right)$.

This implies that as $A_{i}$ increases, $P_{i}\left(X^{*} ; A_{i}\right)$ increases. That is, maximizing $A_{i}$ achieves the maximum winning probability for group $i$

\section{Appendix B}

Here, we repeat our analysis by using the Epstein and Mealem's generalized Tullock contest, and show that Lemma 1 and Proposition 1 hold. We confirm this first. The expected payoff of member $j$ in group $i$ is $U_{i j}=\frac{\sum_{j=1}^{n_{i}} e_{i j}^{r}}{\sum_{j=1}^{n_{i}} e_{i j}^{r}+X_{-i}} a_{i j}-\frac{1}{\beta} e_{i j}^{\beta}$. The first order condition is

$$
\frac{\partial U_{i j}}{\partial e_{i j}}=\frac{r e_{i j}^{r-1} X_{-i}}{\left(\sum_{j=1}^{n_{i}} e_{i j}^{r}+X_{-i}\right)^{2}} a_{i j}-e_{i j}^{\beta-1}=0 .
$$

This can be rewritten as

$$
P_{i}\left(1-P_{i}\right) \frac{r e_{i j}^{r}}{\sum_{j=1}^{n_{i}} e_{i j}^{r}} a_{i j}-e_{i j}^{\beta}=0 .
$$

We process a procedure similar to the one at the end of Section 2 and get $e_{i j}^{r}=\left(\frac{r P_{i}\left(1-P_{i}\right)}{X_{i}}\right)^{\frac{r}{\beta-r}} a_{i j}^{\frac{r}{\beta-r}}$ from (13). By summing up each $e_{i j}^{r}$, we have $\sum_{j=1}^{n_{i}} e_{i j}^{r}=X_{i}=\left(\frac{r P_{i}\left(1-P_{i}\right)}{X_{i}}\right)^{\frac{r}{\beta-r}} \hat{A}_{i}$ where $\hat{A}_{i}=\sum_{j=1}^{n_{i}} a_{i j}^{\frac{r}{\beta-r}}$. Let $\hat{\phi}\left(X_{i}, \hat{A}_{i}\right)=X_{i}-\left(\frac{r P_{i}\left(1-P_{i}\right)}{X_{i}}\right)^{\frac{r}{\beta-r}} \hat{A}_{i}=0$. By differentiating $\hat{\phi}$ with respect to $\hat{A}_{i}$ and noting that $P_{i}$ is a function of $X_{i}$, we have

$$
\frac{d X_{i}}{d \hat{A}_{i}}=-\frac{\hat{\phi}_{\hat{A}_{i}}}{\hat{\phi}_{X_{i}}}=\frac{\left(r P_{i}\left(1-P_{i}\right) / X_{i}\right)^{\frac{r}{\beta-r}}}{\left(\beta-r+2 r P_{i}\right) /(\beta-r)}>0
$$

for $\beta>r$ by using $\hat{A}_{i}=X_{i}\left(\frac{r P_{i}\left(1-P_{i}\right)}{X_{i}}\right)^{-\frac{r}{\beta-r}}$. Therefore, since Lemma 1 holds, Proposition 1 also holds in this case. Proposition 2 holds as well. However, Proposition 3 does not hold. 
We check this second. Under the egalitarian rule, since $X_{i}=\sum_{j=1}^{n_{i}} e_{i j}^{r}=n_{i} e_{i}^{r}$, we have $e_{i}=$ $n_{i}^{-\frac{2}{\beta}} r^{\frac{1}{\beta}} P_{i E}^{\frac{1}{\beta}}\left(1-P_{i E}\right)^{\frac{1}{\beta}}$ from (13). Using this, we have

$$
P_{i E}=\frac{n_{i} e_{i}^{r}}{n_{i} e_{i}^{r}+X_{-i}}=\frac{n_{i}^{\frac{\beta-2 r}{\beta}} r^{\frac{r}{\beta}} P_{i E}^{\frac{r}{\beta}}\left(1-P_{i E}\right)^{\frac{r}{\beta}}}{n_{i}^{\frac{\beta-2 r}{\beta}} r^{\frac{r}{\beta}} P_{i E}^{\frac{r}{\beta}}\left(1-P_{i E}\right)^{\frac{r}{\beta}}+X_{-i}}
$$

and

$$
U_{i E}=P_{i E} \frac{1}{n_{i}}-\frac{1}{\beta} e_{i}^{\beta}=\frac{P_{i E}}{n_{i}}\left(1-\frac{1}{\beta}\left(1-P_{i E}\right) \frac{r}{n_{i}}\right)
$$

Let $\hat{\Delta} \equiv n_{i}^{-\frac{\beta-2 r}{\beta}}$ in relation to $n_{i}$ in (14). We process the same procedure as in the proof of Lemma 2. Rewriting (14), we have

$$
r^{\frac{r}{\beta}}\left(1-P_{i E}\right)\left(P_{i E}\left(1-P_{i E}\right)\right)^{\frac{r}{\beta}}=P_{i E} X_{-i} \hat{\Delta}
$$

By totally differentiating the above expression and conducting the same operations as the proof of Lemma 2, we obtain

$$
\frac{d P_{i E}}{d \hat{\Delta}}=\frac{P_{i E}\left(1-P_{i E}\right)}{\hat{\Delta}\left[-1+\frac{r}{\beta}\left(1-2 P_{i E}\right)\right]}<0
$$

because of $\frac{r}{\beta}\left(1-2 P_{i E}\right)<1$. Differentiating $\hat{\Delta}$ with respect to $n_{i}$, we have

$$
\frac{d \hat{\Delta}}{d n_{i}}=-\frac{\beta-2 r}{\beta} \times \frac{\hat{\Delta}}{n_{i}} .
$$

We then obtain $\frac{d P_{i E}}{d n_{i}}=\frac{2 r-\beta}{n_{i}} \frac{P_{i E}\left(1-P_{i E}\right)}{r\left(1-2 P_{i E}\right)-\beta}$. The sign of this formula depends only on the sign of $2 r-\beta$, as well as (6) from Lemma 2. Therefore, Proposition 2 holds in this case.

By processing the same procedure as the proof of Lemma 4, we obtain

$$
\begin{aligned}
U_{i E}\left(n_{i}, \frac{\beta}{2}\right)-U_{i M} & =P_{i M}\left[-1+\frac{1}{\beta}\left(1-P_{i M}\right) r+\frac{1}{n_{i}}\left(1-\frac{1}{\beta}\left(1-P_{i M}\right) \frac{r}{n_{i}}\right)\right] \\
& =P_{i M}\left(1-\frac{1}{n_{i}^{2}}\right)\left[-\frac{n_{i}}{n_{i}+1}+\frac{r}{\beta}-\frac{r}{\beta} P_{i M}\right] \\
& =P_{i M}\left(1-\frac{1}{n_{i}^{2}}\right)\left[-\frac{n_{i}}{n_{i}+1}+\frac{1}{2}-\frac{1}{2} P_{i M}\right]
\end{aligned}
$$

at $r=\frac{\beta}{2}$. For the above expression to be positive, the sign in the brackets needs to be positive. Thus,

$$
P_{i M}<\frac{1-n_{i}}{n_{i}+1}<0
$$


However, this condition contradicts the definition of the probability. Lemma 4 does not hold. Therefore, Proposition 3 also fails to hold in the generalized Tullock contest.

\section{References}

[1] Baik, K.H. (2008), "Contest with group-specific public-good prizes," Social Choice and Welfare 30: $103-117$.

[2] Cheikbossian, G. and Fayat, R. (2018), "Group size, collective action and complementarities in efforts," Economics Letters 168: 77 - 81.

[3] Choi, J. P., Chowdhury, S.M., and Kim, J. (2016), "Group contests with internal conflict and power asymmetry," Scandinavian Journal of Ecnomics 118: 816 - 840.

[4] Cornes, R. and Hartley, R. (2005), "Asymmetric contests with general technologies," Economic Theory 26-4: $923-946$.

[5] Crutzen, B. S., Flamand, S., and Sahuguet, N. (2020), "A model of a team contest, with an application to incentives under list proportional representation," Journal of Public Economics 182: 104109.

[6] Epstein, G.S. and Mealem, Y. (2009), "Group specific public goods, orchestration of interest groups with free riding," Public Choice 139 3-4: 357-369.

[7] Esteban, J. and Ray, D. (2001), "Collective action and the group size paradox," American Political Science Review 95 - 3: 663 - 672.

[8] Kolmar, M. and Rommeswinkel, H. (2013), "Contests with group-specific public goods and complementarities in efforts," Journal of Economic Behavior and Organization 89: 9-22. 
[9] Nitzan, S. and Ueda, K. (2011), "Prize sharing in collective contests," European Economic Review 55: 678-687.

[10] Nitzan, S. and Ueda, K. (2014), "Intra-group heterogeneity in collective contests," Social Choice and Welfare 43: $219-238$.

[11] Olson, M. (1965), The logic of collective action, Harvard University Press, Cambridge, MA, US.

[12] Tullock, G. (1980), "Efficient rent seeking," Buchanan, J.M., Tollison, R.D., and Tullock, G. (Eds.), Toward a theory of the rent-seeking society, Texas A\&M University Press, College Station, TX, US: 97-112.

[13] Ueda, K. (2002), "Oligopolization in collective rent-seeking," Social Choice and Welfare 19-3: $613-626$. 


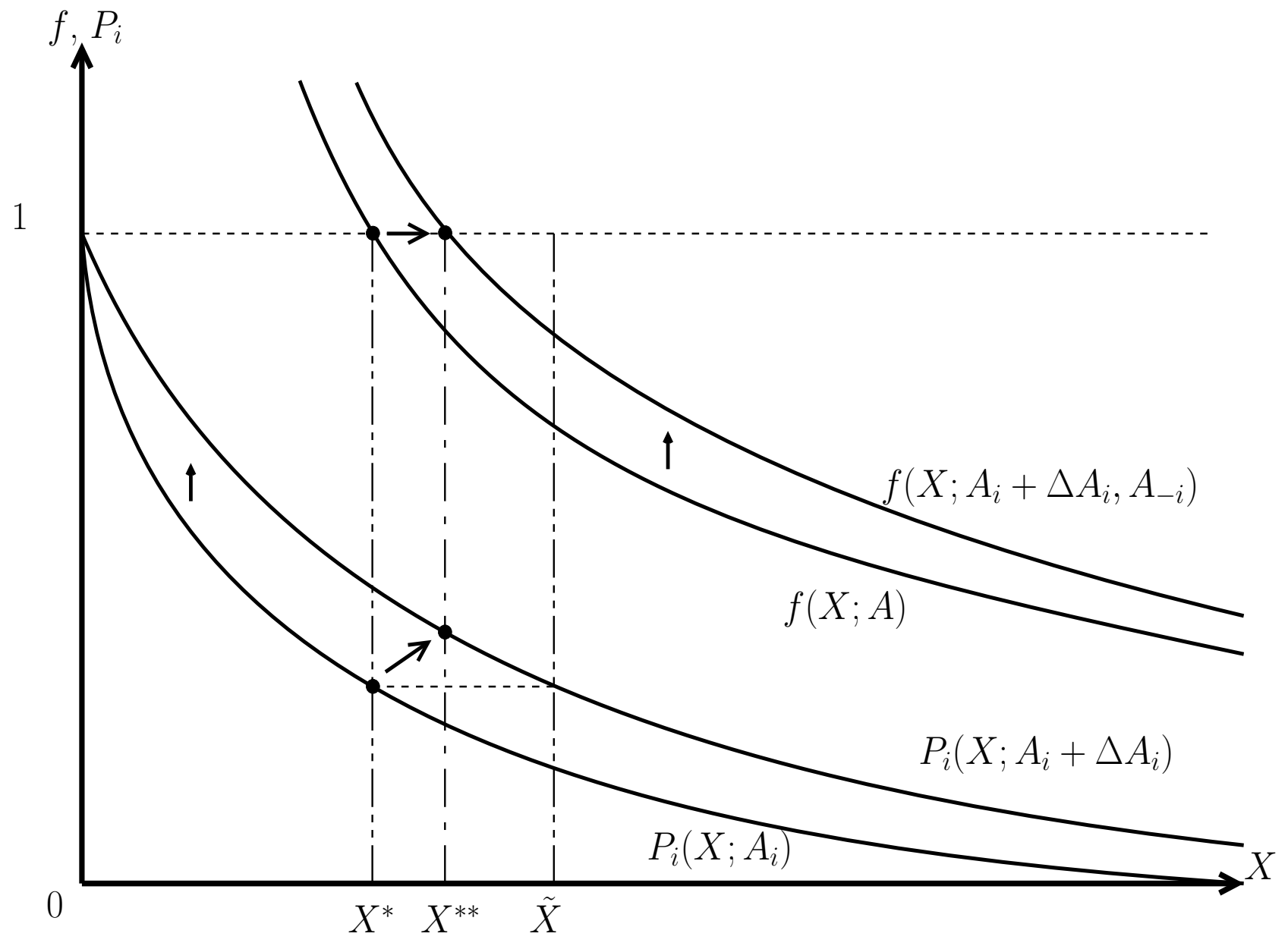

Figure 1: Share function $P_{i}\left(X ; A_{i}\right)$ of group $i$ and aggregate share function $f(X ; A)$ 